\title{
Photo-graphies
}

\section{Photographier James Joyce}

Le portrait de l'écrivain par Gisèle Freund

\section{Monique Sicard}

\section{(2) OpenEdition}

Journals

\section{Édition électronique}

URL : http://journals.openedition.org/genesis/1475

DOI : 10.4000/genesis. 1475

ISSN : 2268-1590

Éditeur :

Presses universitaires de Paris Sorbonne (PUPS), Société internationale de génétique artistique littéraire et scientifique (SIGALES)

\section{Édition imprimée}

Date de publication : 15 avril 2015

Pagination : 117-129

ISBN : 978-2-84050-992-9

ISSN : 1167-5101

\section{Référence électronique}

Monique Sicard, «Photographier James Joyce », Genesis [En ligne], 40 | 2015, mis en ligne le 03 avril 2017, consulté le 16 mai 2019. URL : http://journals.openedition.org/genesis/1475; DOI : 10.4000/ genesis. 1475 


\title{
Photographier James Joyce Le portrait de l'écrivain par Gisèle Freund
}

\author{
Présenté par Monique Sicard
}

\section{Contexte théorique}

\begin{abstract}
Chaque moment de l'histoire voit naître des modes d'expression artistique particuliers, correspondant au caractère politique, aux manières de penser et aux goûts de l'époque. Le goût n'est pas une manifestation inexplicable de la nature humaine, il se forme en fonction des conditions de vie bien définies qui caractérisent la structure sociale à chaque étape de son évolution.
\end{abstract}

La phrase introductive du livre Photographie et société 1 écrit par Gisèle Freund guide l'analyse critique des œuvres d'une photographe passionnée par la réalisation des portraits des écrivains, ses contemporains. En référence à l'explosion de la demande de portraits sous le Second Empire, elle écrit :

Le portrait photographique correspond à un stade particulier de l'évolution sociale : l'ascension de larges couches de la société vers une plus grande signification politique et sociale ${ }^{2}$.

La thèse qu'elle soutient en 1936, "La photographie en France au XIX ${ }^{e}$ siècle », est la première jamais consacrée à l'histoire de la photographie. Elle n'est pas encore achevée quand l'étudiante en sociologie et histoire de l'art entreprend son vaste projet de collecte de visages d'écrivains. Le premier est celui d'André Malraux, en 1935. Le tour de James Joyce arrive en 1938 ; Gisèle Freund le photographie de nouveau en 1939. Nul doute qu'elle a alors en tête une analyse du milieu littéraire parisien. Elle affirme cependant ne réaliser de portraits d'écrivains que de ceux dont elle apprécie les œuvres.
Par de nombreux aspects, son travail opère une liaison entre les mots et les images, l'écrivain et le photographe, l'écrivain et sa photographie. Cette œuvre double se nourrit d'une réflexion théorique profonde sur l'enjeu photographique. À ce titre, elle fournit des éléments d'analyse, non seulement pour elle-même, mais aussi pour toute œuvre photographique depuis l'invention de la photo, dans la première moitié du XIX ${ }^{\mathrm{e}}$ siècle.

Gisèle Freund, qui côtoie Walter Benjamin à Paris, de 1933 à 1940, a clairement conscience des liens qui unissent le développement industriel et l'esthétique. Comme Benjamin, elle s'intéresse à la perte d'unicité et d'aura de l'œuvre d'art avec le développement de la photographie. Mais peut-être mieux que lui, car elle raisonne en professionnelle, elle a conscience de l'importance des dispositifs de prise de vue, des phases préparatoires aux portraits et de leur nature "fabriquée ». Tous deux ont le sentiment que l'expansion de la photographie puis sa banalisation, voire son déclin symbolique au cours du $\mathrm{xx}^{\mathrm{e}}$ siècle, sont demeurés inaperçus, malgré l'ampleur des enjeux dont ils sont porteurs. Chacun d'eux a à cœur d'écrire sur la photographie.

\section{Le dossier génétique des portraits de James Joyce par Gisèle Freund}

Le dossier génétique comporte tant des textes que des objets photographiques. Au titre des textes : un carnet

1. Gisèle Freund, Photographie et société, Paris, Éditions du Seuil, coll. « Histoire », 1974, p. 5.

2. Ibid., p. 11 
répertoire de terrain, des brouillons manuscrits et tapuscrits constituant le « journal personnel ». Au titre des objets photographiques : des négatifs $24 \times 36$, des planchescontacts, des diapositives couleur (positifs directs), des tirages de presse, des tirages d'exposition de grand format. Parmi ceux-ci, les négatifs et les diapositives sont des objets uniques, dits «non reproductibles ».

Les archives de la photographe déposées à l'IMEC comprennent mille six cents négatifs noir et blanc et les planches-contacts correspondantes, huit mille deux cents diapositives (duplicata et originaux confondus), mille contretypes et plus de huit mille tirages de presse noir et blanc. Sur ces mille six cents négatifs, plus d'une centaine réfèrent au portrait de James Joyce. Les trois planchescontacts noir et blanc qui leur correspondent sont des pièces importantes du dossier génétique. Au sein du dossier personnel, huit brouillons tapuscrits avec des annotations manuscrites à l'encre relatent les séances de pose réalisées avec James Joyce. Chaque élément du dossier apporte son lot d'informations relatives à la réalisation des portraits.

Le répertoire alphabétique de 1938-1939 (fig. 1 à 3) propose, jeté à la hâte sur le papier, un témoignage écrit sur le vif de scènes, vécues comme extraordinaires, ayant conduit aux prises de vue.

Le journal personnel offre accès à des commentaires raturés, recopiés, plusieurs fois repris, enrichis, modifiés (fig. 4). Il s'agit d'analyses synthétiques rédigées peu de temps après les séances de pose. À la fois sensibles et précises, elles témoignent des relations qui se nouent entre la photographe et ses modèles ; le regard observateur de Gisèle Freund scrute les détails sociologiquement signifiants ou formellement intéressants.

Les planches-contacts (fig. 5) rendent compte des démarches de l'artiste, de ses déplacements, de sa mobilité lors des plongées et contre-plongées, enfin des changements de diaphragmes, de focale, de cadrage mais aussi des sélections ultérieures effectuées au moment du tirage, des recadrages des négatifs sélectionnés.

\section{Le répertoire}

Le répertoire alphabétique, pièce importante du dossier génétique, mérite une attention particulière.
Il est muni d'une couverture marron en carton rigide (environ $22 \times 15 \mathrm{~cm}$ ) sur laquelle est collée une étiquette professionnelle, « Photo Gisèle Freund 18 rue de l'Odéon, Paris-6 ». Une seconde étiquette fait savoir que le carnet a été acheté à deux pas de là, chez Roques \& fils, 113 rue de Condé, 4 rue de l'Odéon Paris VIe. Sur les deux dernières pages du carnet sont collées les indications tarifaires détaillées des séances de pose (à domicile ou chez la photographe) et des tirages photographiques noir et blanc ou couleur. Le tirage couleur valait alors six à sept fois plus cher que le tirage noir et blanc.

Ainsi, c'est bien en professionnelle que Gisèle Freund se lance à Paris, en 1938, deux ans après avoir soutenu sa thèse, trois ans après avoir fui l'Allemagne nazie.

À chaque lettre du répertoire alphabétique, est soigneusement noté à l'encre noire le nom de ceux pour lesquels une séance de pose a été réalisée, soit plus d'une centaine de personnes, pour la plupart écrivains.

Chacun de leurs noms est suivi d'une adresse et d'un numéro de téléphone. Sont également notés avec précision la date de la prise de vue, le numéro des rouleaux de pellicules utilisés. Suivent, au crayon mine, des commentaires souvent très personnels de la photographe sur la personne rencontrée. Ainsi sont répertoriés André Breton, Roger Caillois, Paul Claudel, Léon-Paul Fargues, François Mauriac, Paul Nizan, André Gide, Elsa Triolet, Louis Aragon, Paul Valéry et bien d'autres...

Sous le nom d'André Breton, il est écrit : «Pape du surréalisme ». Sous celui de Georges Duhamel : «Vient avec tout un choix de cravates ». Sous celui de Paul Éluard : «Grand poète ».

Ces propos crayonnés sont souvent brefs, voire laconiques, mais au nom de James Joyce (fig. 1 à 3) le texte est nourri. Occupant cinq pages du carnet, mêlant l'anglais et le français, il ne s'arrête qu'à la lettre K, laissant place à Arthur Koestler.

Le nombre élevé d'écrivains photographiés - plus d'une centaine - durant deux années, de 1938 au tout début de 1940, apparaît extraordinaire. Il témoigne que Gisèle Freund, qui vivait à Paris de sa photographie, faisait preuve d'un dynamisme caractéristique du milieu des émigrés. Cinq ans après son arrivée, elle était donc déjà bien installée au cœur du quartier latin, au sein du milieu littéraire parisien. 
Le carnet répertoire montre l'émergence avant-guerre d'un projet photographique solidement construit. Il offre en outre des pistes génétiques utiles à la compréhension des dispositifs photographiques et des intentions de l'auteur. Il est enfin révélateur du regard porté par l'artiste sur le monde littéraire parisien. Il avait certainement une fonction d'aide-mémoire, tant pour la photographe que pour l'écrivain qu'était Gisèle Freund. Il est possible qu'elle ait eu d'emblée en tête l'écriture d'un futur ouvrage 3 .

\section{Rencontre et premières prises de vue}

Gisèle Freund raconte dans son journal, et dans différents récits, comment elle fit la connaissance de Joyce. À l'époque, les thèses des étudiants devaient être publiées, mais Gaston Gallimard avait refusé celle de la photographe car « il ne s'agissait pas de littérature ». Gisèle Freund s'était alors tournée vers Adrienne Monnier, toujours prête à aider les femmes. Elle se trouva donc avoir le même éditeur que James Joyce, qu'elle considérait comme l' « une des idoles littéraires de sa génération ${ }^{4}$ ». Gisèle Freund avait lu Ulysse, que lui avait offert Adrienne Monnier. En 1936, elle rencontre Joyce au cours d'un dîner organisé par l'éditrice. Il était alors accompagné de sa femme Nora. La photographe raconte qu'à cette occasion naquit chez elle l'envie de faire le portrait de l'écrivain : « en observant les ombres et les lumières qui modelaient son visage 5 ». Au cours des mois suivants, elle tente en vain de le convaincre de se laisser photographier. Joyce refuse longtemps, sous divers prétextes. Ce n'est qu'au printemps 1938 que furent réalisés les portraits en noir et blanc.

Gisèle Freund note dans son journal :

Je dois aller chez lui le lendemain à $11 \mathrm{~h}$ pour la première séance. Très bourgeois. Il habite le 7ème arrondissement. [...] Je mets mon petit Leica dans mon sac et deux ampoules que je mettrai dans les lampes au cas où la lumière laisserait à désirer et sonne à la porte à l'heure indiquée. Il m'amène dans un salon très conventionnellement meublé. Joyce me présente à Eugène Jolas : «Entrez, on vous attend ».

La photographe décrira un homme très grand et mince, déjà un peu voûté, animé de mouvements lents et incertains, « sans doute à cause de ses yeux. Il marchait en tâtonnant 6 ». Eugène Jolas, écrivain, traducteur, critique littéraire et ami de Joyce est présent.

Gisèle Freund donne ses consignes à Joyce et Jolas (fig. 4) :

Asseyez-vous comme vous êtes habitués à le faire en travaillant ensemble. Alors Joyce se laisse tomber dans un fauteuil en cuir et Jolas s'asseoit sur le canapé à son côté. <Sur une chaise $>$, [sur] une table il y a un monton ${ }^{7}$ de feuilles $<$ d'épreuves $>$, ce sont les épreuves de Finnegan's wake et Jolas commence à lire un passage à haute voix.

La pièce est claire mais le film que j'utilise n'est pas très fort. Je peux pourtant faire des expositions d'une vi trentième de seconde ${ }^{8}$ en ouvrant tout grand mon objectif. Je perdrais en profondeur de champ mais j'aurais des photos vivantes?.

Ainsi témoigne Gisèle Freund dans son journal personnel :

Je n'ai jamais indiqué à mes modèles la moindre pose ; j'ai toujours essayé de leur faire parler de leurs propres problèmes, sachant qu'ils oublieraient l'appareil braqué en face d'eux.

Grâce à l'ensemble de ces documents textuels et photographiques, nous savons que les portraits en noir et blanc de James Joyce ont été réalisés sur trois journées, au printemps 1938 .

La première journée de prise de vue en noir et blanc eut lieu en compagnie d'Eugène Jolas, dans l'appartement parisien de Joyce, rue Edmond-Valentin, dans le VIIe arrondissement. La deuxième journée s'est déroulée dans la librairie Shakespeare and Co, rue de l'Odéon, avec Sylvia Beach et Adrienne Monnier, éditrices respectivement des versions anglaise et française d'Ulysse. La troisième journée rend compte de la visite de Joyce à son fils Giorgio, son petit-fils Stephen, sa belle-fille Lucia, dans leur jardin et leur maison de la rue Scheffer, à Paris.

3. Nous tenons à remercier Daniel Ferrer et Lydie Rauzier pour leur lecture attentive du carnet répertoire de Gisèle Freund.

4. Gisèle Freund, Dossier gris « Journal personnel », Notes manuscrites, s.d., IMEC [désormais « Journal personnel »].

5. « Journal personnel».

6. «Journal personnel».

7. Certainement issu de l'espagnol montón (tas).

8. Soit « à la vitesse d'un trentième de seconde ».

9. « Journal personnel». 
Nous savons que ces portraits de Joyce ont été réalisés avant tout «par plaisir10 » et non sous la contrainte d'une commande. Plus tard cependant, alors que la parution de Finnegans Wake avait été retardée d'une année, le Time se dira intéressé par un portrait en couleurs de l'écrivain pour sa couverture. Les prises de vue des 8 et 9 mars 1939 répondent à cette commande.

Le carnet répertoire rend compte des incidents qui ont émaillé les prises de vue en couleurs des 8 et 9 mars 1939. Puis, comme un post-scriptum rétrospectif, Gisèle Freund relate la rencontre qui avait eu lieu une année plus tôt chez Giorgio ; il s'agissait alors de la troisième journée de prise de vue en noir et blanc. Cet ajout est d'ailleurs séparé par un trait du récit des journées des 8 et 9 mars 1939 et précédé de la mention « $\underline{38} »$ [1938] (fig. 2).

\section{La planche-contact de la seconde journée de prise de vue en noir et blanc, en 1938}

Posés dans la chambre noire sur le papier sensible, les négatifs fournissent «par contact» de petits positifs. Plus lisibles, ceux-ci permettent d'évaluer les photographies à tirer, celles qui devraient être abandonnées, les recadrages à effectuer, les techniques de tirage à adopter.

En tirant simultanément tous les négatifs d'une pellicule, Gisèle Freund, de nouveau, est en avance sur ses contemporains. En effet, il s'agissait plutôt à l'époque d'effectuer de petits tirages papier individuels à partir de négatifs dûment sélectionnés issus de la pellicule exposée. La réalisation d'une planche complète, par contact, n'est apparue que progressivement, dans un second temps. La technique s'est surtout développée après guerre.

La planche-contact archivée à l'IMEC sous le numéro 19 (fig. 5) témoigne des prises de vue effectuées en 1938, le second jour, dans la rue, lors de l'arrivée de Joyce à la librairie Shakespeare and Co et au sein même de cette librairie. L'écrivain se trouve en situation de travail en compagnie de Sylvia Beach et d'Adrienne Monnier.

Cette planche, constituée à la hâte, de façon non systématique, provient d'une pellicule de trente-six poses de format $24 \times 36 \mathrm{~mm}$, réalisée au Leica (contraction de
Leitz camera), comme en témoigne Gisèle Freund dans ses manuscrits.

Dans ses « Souvenirs de Berlin11 », elle rappelle les conditions d'acquisition de ce Leica :

J'allais encore à l'école lorsque je demandais à mon père de m'offrir un appareil photo. Il me donna un Voigtländer. Déjà enfant, je ressentais le besoin de m'exprimer et la photo comblait mon désir. Après mon baccalauréat, je lui demandais un Leica. Ce petit appareil maniable, que des millions de photographes amateurs et professionnels utilisent aujourd'hui, était encore presque inconnu à l'époque. J'étais loin de me douter que la photographie allait devenir un jour mon métier. Mais je m'intéressais avant tout à la sociologie et à l'histoire de l'art. C'est ainsi que je partis à Fribourg, puis à l'université Goethe de Francfort, où enseignait le sociologue Karl Mannheim.

Leica a adapté le petit format à la photographie. Ces appareils légers, robustes, aux objectifs clairs, permettent une grande mobilité du photographe qui n'hésite pas dès lors à s'approcher, s'éloigner de son sujet, voire à tourner vivement autour de lui. De même que le Voigtländer, premier appareil possédé par Gisèle Freund, le Leica est très adapté à la réalisation de portraits. Bénéficiant d'objectifs interchangeables, il oblige le photographe à des manipulations rapides. Nous devons ainsi imaginer Gisèle Freund œuvrant avec vivacité, attentive à utiliser la meilleure focale, à capter les plus belles lumières, à choisir le cadre le mieux adapté, à saisir les expressions les plus significatives de son modèle.

La planche-contact $n^{\circ} 19$ comporte ainsi trente-deux images. Le négatif de trente-six poses fut coupé en six bandes de six poses chacune. Quatre de ces bandes furent conservées dans leur intégralité. Des deux autres bandes furent extraits des morceaux d'une, deux et trois images. Sur les trente-six images que devait comporter le négatif d'origine, quatre ont donc été jugées inintéressantes par la photographe.

La première ligne comporte une bande de trois images présentées haut et bas inversés, réalisées dans la librairie

10. «Journal personnel».

11. Tapuscrit IMEC, ancienne cote B2. 
Shakesperare and Co, dont la dernière est sous-exposée. Cette bande rend compte du dialogue professionnel entre Sylvia Beach, Adrienne Monnier et James Joyce. La photographe s'est progressivement rapprochée de son sujet, jusqu'à ne plus cadrer que Joyce et Sylvia Beach, puis Joyce seul. La dernière image est un beau portrait de l'écrivain cadré verticalement, portant sa main gauche à la bouche, capté devant une étagère de livres. L'image fut retenue pour le tirage.

La deuxième ligne est constituée de six images consécutives, présentées inversées haut et bas, comme les précédentes, cette fois toutes cadrées verticalement. Il ne s'agit pas de cerner des personnages dans un décor, mais bien de capter à son insu un homme seul. Les photos montrent l'arrivée de Joyce en taxi. Descendu de voiture, il se rapproche du conducteur afin peut-être de payer la course, puis s'éloigne. La photographe, elle, est mobile, elle se rapproche de l'écrivain jusqu'à le cadrer plein pied, puis s'en va lorsque lui-même se dirige vers la librairie. La dernière image montre Joyce accompagné d'Adrienne Monnier venue l'accueillir. La photographe s'est éloignée pour les saisir tous les deux dans un cadre large et certainement se faire plus discrète. Cette dernière image est recadrée au crayon gras rouge, pour tirage.

La troisième ligne est formée de cinq images : une image seule et deux séquences de deux images chacune sont présentées dans une chronologie bousculée. La première photographie, isolée, montre l'intérieur de la librairie avec les trois protagonistes, James Joyce, Sylvia Beach, Adrienne Monnier. Suivent deux images cadrées verticalement. Antérieures aux précédentes, elles montrent Joyce accompagné d'Adrienne Monnier arrivant à la librairie. La photographe a réalisé ces images à très vive allure, comme le montre leur faible intervalle de succession. Les deux dernières images de cette troisième ligne sont elles aussi cadrées verticalement : réalisées à l'intérieur de la librairie, elles montrent Joyce bavardant avec Sylvia Beach.

La quatrième ligne rassemble six images des trois personnes discutant à l'intérieur de la librairie. Fruit d'une erreur de la part de la photographe, le négatif a été posé en miroir, face sensible sur le dessus et non plaqué contre le papier, comme il aurait dû l'être lors du tirage de la planche-contact. La lecture de ces six images en est alors rendue délicate ; elle exige une inversion droite-gauche. Les observer dans un miroir facilite la compréhension du dispositif spatial. Cette hypothèse d'une inversion résulte de la comparaison de ces images avec d'autres images de la même planche-contact, mais aussi avec les tirages positifs effectués du vivant de Gisèle Freund et sous son contrôle. Comme l'indiquent les annotations au crayon gras, quatre photographies au moins appartenant à cette ligne ont été retenues pour tirage.

La cinquième ligne, constituée de six images successives cadrées horizontalement, présente des scènes similaires. Elle aussi inversée, elle doit être lue en miroir. Deux des photographies, sous-exposées, sont illisibles. Lors de toutes les prises de vue d'intérieur, la photographe a modifié son cadre, ses réglages. Elle s'approche ou se recule. Ici, jusqu'à prendre une vue en plongée du groupe de travail, alors qu'elle est montée sur un quelconque support, peut-être une marche d'escalier.

Un tirage de la photographie $n^{\circ} 5$ est reproduit dans cet article (fig. 6). Il apporte la preuve d'une inversion en miroir effectuée par mégarde des deux bandes contacts des lignes 4 et 5 .

La sixième ligne offre, en six images consécutives, le passage d'un cadre horizontal à un cadre vertical. La photographe, en effet, cadre d'abord le couple formé par l'écrivain et Sylvia Beach avant de se concentrer, en cadre vertical, sur Joyce seul, portant sa veste noire ${ }^{12}$ et un nœud papillon. Suivent ainsi quatre portraits de l'écrivain sur fond d'une étagère remplie de livres.

Mais Gisèle Freund ne cesse de se mouvoir : le portrait se resserre peu à peu. Au cadre " genoux »succède un cadre " taille», puis un cadre « épaules ». Le regard dirigé vers la droite en direction de ses deux collaboratrices, Joyce met sa main gauche, puis ses deux mains, devant la bouche, appuie sa tête sur cette même main gauche. La dernière image est un portrait classique, centré sur le seul visage de l'écrivain.

12. L'information émane des contretypes couleur effectués d'après les diapositives. Le premier jour, Joyce revêt une veste d'intérieur rouge ; le second, une veste noire. Le carnet répertoire de terrain fournit également des informations sur cette question. 
L'ensemble de la planche-contact témoigne de la hâte et du désordre relatif avec lesquels travaillait Gisèle Freund. Une flèche tracée au crayon gras rouge indique les inversions à effectuer pour le tirage. Les images dont les numéros sont entourés ont été retenues pour un tirage, mais rien n'indique que ce marquage ait été systématique. L'une des photographies réalisées dans la rue de l'Odéon et montrant Joyce en compagnie d'Adrienne Monnier propose, au crayon gras rouge, un recadrage recentrant l'attention sur les personnages eux-mêmes.

D'une manière générale, Gisèle Freund se montre très mobile, se déplaçant et recadrant sans cesse ses images, passant du cadre vertical au cadre horizontal et inversement. Le plus souvent, elle choisit un sujet, préserve un cadre large puis n'a de cesse que de se rapprocher. À aucun moment Joyce ne tourne la tête vers elle et ne semble prendre en compte sa présence.

Cette planche-contact et les tirages de presse correspondants montrent que les portraits en noir et blanc de Joyce relèvent de deux statuts différents. Les unes furent le fruit d'une sollicitation officielle de l'écrivain par la photographe. Les autres, qui tiennent d'une pratique de paparazzi attentif aux gestes les plus anodins des «people », n'ont pas obtenu l'accord de Joyce. Les premières ont donné lieu à des cadrages précis, de nombreux essais et prises de vue effectués dans le calme. Il en est ainsi des photographies réalisées dans la librairie Shakespeare and Co.

Il faut compter parmi les secondes le voyage en taxi du second jour et l'arrivée à la librairie en compagnie d'Adrienne Monnier. Sur la planche-contact, le recadrage au crayon gras rouge peut être interprété comme un signe de la hâte avec laquelle s'est effectuée la prise de vue. Sur un certain nombre de photographies, l'imprécision de la mise au point rend compte de la même relation entre une photographe au regard affiné saisissant hâtivement les gestes d'un homme très mal voyant, ignorant sa présence.

Le troisième jour des prises de vue en noir et blanc l'image de Joyce s'arrêtant chez un fleuriste pour garnir sa boutonnière d'un œillet avant la visite à son fils -, les cadrages incertains signent des images réalisées sans l'accord de leurs protagonistes. Mais la photographie du chien Schiap tenté par le sucre du jeune Stephen et qui se dresse sur son arrière-train face à la photographe a été réalisée à la volée, comme en témoigne la mauvaise qualité du cadrage, cependant sans que la photographe se cache.

La photographe notera dans son journal personnel que toutes les photographies réalisées ont été montrées à Joyce, sauf celles où il sort du taxi13. Preuve, s'il y avait encore besoin de le souligner, que des images furent « volées » par une immense admiratrice à un écrivain presque aveugle qui ne vit rien de ces prises de vue à la sauvette.

\section{Les portraits en couleurs de mars 1939}

Les portraits en couleurs réalisés en mars 1939 placent Gisèle Freund au premier rang de la modernité. Elle écrira dans Photographie et société :

Le film en couleurs pour amateurs est d'histoire récente. Ce n'est qu'en 1937 que Kodak vendit pour la première fois le Kodachrome et Agfa l'Agfacolor. Très peu d'amateurs les utilisaient à cette époque, car les films étaient beaucoup plus chers que la pellicule noire. Il fallait acheter en plus un projecteur pour les visionner, car c'étaient des diapositives. Les reproductions en couleurs sur papier coûtaient un prix exorbitant et ne se faisaient qu'en Amérique et en Angleterre. À quelques rares exceptions près, les professionnels non plus ne faisaient pas de couleur, car la plupart des revues ne possédaient pas encore les machines nécessaires pour l'imprimer $^{14}$.

Ces pellicules couleur étaient moins sensibles que le noir et blanc : les prises de vue nécessitaient l'usage d'éclairages supplémentaires. Ce fut le cas pour les portraits de Joyce. Craignant pour ses yeux, il avait demandé à la photographe de ne pas utiliser de lumière vive, mais elle est parvenue à ses fins. Deux séries de prises de vue en couleurs eurent ainsi lieu en complément des séries noir et blanc.

13. Gisèle Freund, Trois Jours avec Joyce, Paris, Denoël, 2006 (1982), p. 43 à 46.

14. Photographie et société, op. cit., p. 197. 
Finnegans Wake est en voie d'achèvement et doit paraître simultanément en Angleterre et aux États-Unis, comme en témoigne Gisèle Freund dans son journal.

Ayant promis à l'écrivain que je ne l'importunerai plus, je répugnais à faire de nouvelles démarches auprès de lui. Mais ses amis pensaient qu'une couverture de revue ferait beaucoup de publicité d'un livre aussi difficile que Finnegan's wake15. Ce fut Sylvia Beach qui trouva l'astuce. Irlandais qui se sentait intimement lié à ses personnages romanesques, Joyce était aussi très superstitieux. Or il se trouvait que le nom de mon mari était le même qu'un des héros d'Ulysse 16 .

Gisèle Freund écrivit à Joyce en évoquant l'impact international des portraits qu'elle réalisait. Blum, nom de son mari, servit d'homonyme à celui de Léopold Bloom, personnage principal d'Ulysse. Simultanément, Gisèle Freund demanda à l'écrivain Louis Gillet d'intervenir auprès de Joyce pour lui expliquer qu'un reportage photographique publié dans différentes revues ne pouvait que soutenir le livre. En retour, Joyce téléphona à Adrienne Monnier en lui demandant que Gisèle vienne le lendemain chez lui organiser une séance de projection de ses photos d'écrivains.

Le 8 mai 1939 Gisèle Freund se rend chez l'écrivain. Joyce se tient à cinquante centimètres de l'écran, son visage presque collé à ceux des écrivains présentés grandeur nature. Lorsque la projection s'achève, Joyce reconnaît la qualité du travail, manifeste son approbation et donne son accord pour une séance de prise de vue en couleurs.

Le carnet répertoire rend compte de cette séance (fig. 1) : «Joyce aime les pix ${ }^{17}$. Se lève - malheur - se cogne la tête contre une lampe - "I' $m$ bleeding. You want to kill $m e "$ [Je suis blessé, vous voulez me tuer]. Se tient la tête. » Gisèle Freund applique une recette héritée de son enfance : elle pose contre la plaie le métal froid d'une paire de ciseaux. Une demi-heure plus tard, elle peut enfin réaliser des portraits en couleurs du grand écrivain.

Dans son journal personnel18, elle raconte plus précisément la séance :

Il avait revêtu une veste d'intérieur rouge et ses longues mains sensibles portaient plusieurs bagues. Il paraissait tout à fait malheureux à l'idée d'être photographié et me jetais des regard inquiets. Sa nervosité me gagnait : je commençais à laisser tomber des objets ; l'atmosphère se tendait de plus en plus. Soudain comme Joyce cherchait à tâtons son chemin vers le fauteuil que j' avais disposé pour lui, sa tête heurta une lampe. Il poussa un cri aigu comme s'il avait été poignardé et se prit le front à deux mains.

Je saigne. Vos sacrées photos seront ma mort ! s'écria-t-il.

Nora, avez-vous des ciseaux? demandais-je à sa femme qui était dans la pièce à côté. Je pressai l'acier froid contre l'égratignure presque imperceptible, afin de prévenir l'enflure, remède dont le souvenir remontait à mon enfance.

Calmé, Joyce s'assit et se mit à étudier la page d'un livre à la loupe, comme il le faisait souvent. J'appuyais sur le déclic et achevai mon film le plus vite possible avant de promettre au patient que cette fois, je ne le dérangerai vraiment plus jamais.

Visiblement soulagé, il me garda encore quelques minutes ; nous parlâmes de Finnegan's Wake, spéculant sur l'accueil de la critique et du public. À la fin, la voix de mon interlocuteur était devenue faible, exténuée ; il parlait de la mort - de sa mort - prédisant que Finnegan's Wake serait son dernier livre.

Je l'assurai qu'après des années de travail intense tous les écrivains sont déprimés, épuisés ; qu'il était encore jeune (il n'avait que 56 ans).

J'appelai un taxi et demandai au chauffeur de m'emmener le plus vite possible au laboratoire où m'attendait un technicien pour développer le film; il fallait que le meilleur portrait de Joyce parte immédiatement au Time Magazine à New York.

Ce jour-là, le 8 mars 1939, Gisèle Freund quitte Joyce à $17 \mathrm{~h}$, s'engouffre dans un taxi afin de déposer au plus vite les pellicules au laboratoire, presse le chauffeur. Hélas, la voiture a un accident. Gisèle Freund pense que ses négatifs sont perdus et téléphone à Joyce («Vous avez jeté un sort à mes photos »). Il lui propose de revenir le lendemain, 9 mars 1939. Ce qu'elle fait. Mais alors l'écrivain a changé d'attitude : il est plus affable, plus conciliant, plus attentif, plus disponible, essaie de luimême différentes poses. Lorsqu'elle arrive au laboratoire, Gisèle Freund a la joie de constater que le film du jour

15. L'orthographe est de Gisèle Freund.

16. «Journal personnel».

17. Pix pour pictures (les images).

18. «Journal personnel », feuillet non encore répertorié, non daté, relatant la séance du 8 mars 1939. 
précédent n'a pas souffert de l'accident. Elle dispose donc de deux séries complètes de photos de l'écrivain.

Plus tard, quand le journal Time paraît avec le portrait de l'écrivain en couverture, Joyce, heureux comme un enfant, montre la revue à tous ceux qui viennent le voir. Mieux encore, s'amusant de cette histoire d'accident de taxi, il conclut devant ses amis : «Je lui avais dit que [je] ne me laisserais jamais photographier en couleurs. Gisèle m'a eu, non pas une fois, mais deux. Elle est plus forte que les Irlandais ${ }^{19}$. »

19. Ces phrases sont extraites du journal personnel. Dans le carnet répertoire, les paroles de Joyce sont rapportées de cette manière (fig. 2) : "I told her I would never be photographed in color. Well Miss. B. caught me twice. She's stronger than the Irish. » 
1neproductirs: coner de Time -8 misi 1939 LLNK

Phruecale from un chambror (Life - Time) to eat colon of Jrace fon Time cover (Finileran'sllatie)

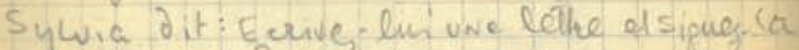

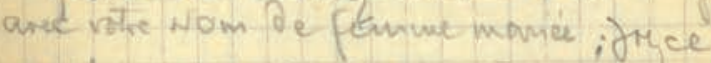
ve refrare pas de e, sisukerstivenxEnvayiletke 6 warn - Rexins le 7- Jiger we repre riven a hime Blum.

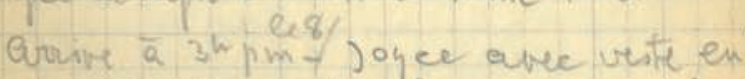

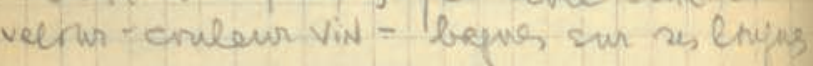
mann - xe soupin hus le tomps.

J'ar apporte wowelles envears plitor ectuvans fléeran a $0.50 \mathrm{~m} \mathrm{re}$

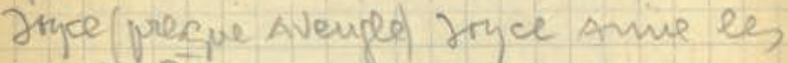
pix. Se leve - malliew - se cogne ra léte conthe une lawpe -

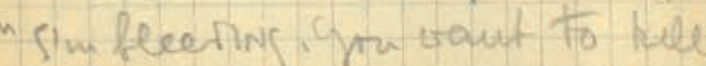

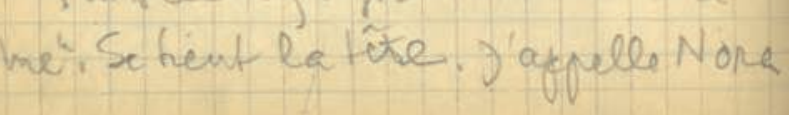

\section{James Joyce}

R.No: 27,28

8-3-1939 chęlui-zeséarce 9-3-1939
Sui etait on côté. Elle srurit (mince Gount-encone bin) Elle apparle ie,

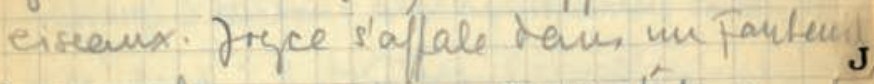

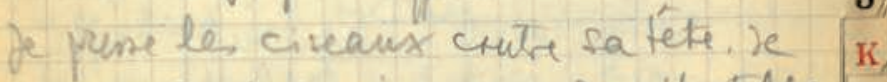
stwpine, Gu'st men. Nora srucit. Elle we le peñ pr. An serrenx

Tais phits 30 minutes fen, FaO Les lumieris le frut smaffin.

He pars a shewre - Taxi-crash -

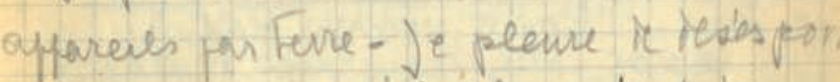
a la maison if telephre trut te subr a pee nivijuce, you damnel we penoforyon put frive kuno of a hal dush spele on then and me tax. crashed S Gan almind bulles and yon puitos are ruined.

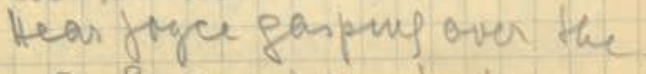

beove. So sivas pight - he hao

Fig. 1 à 3 : Pages « James Joyce » du répertoire alphabétique des portraits réalisés par Gisèle Freund entre 1938 et 1940 (Archives Gisèle Freund/IMEC/Fonds MCC)

1 repr`ducti` $n$ : c`ver de Time -8 mai 1939 ULNK

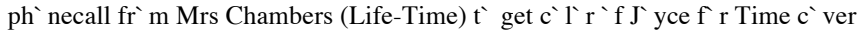
(Finnegan's Wake)

Sylvia dit : Ecrivez-lui une lettre et signez-la avec v` tre $n ` m$ de femme mariée ;

J yce ne refusera pas. Il est si superstitieux. Env`yé lettre le 6 mars. Rép`nse le 7. $\mathrm{J}$ yce ne refuse rien à mme Blum. Arrive à $3 \mathrm{~h} \mathrm{pm}{ }^{\text {le } 8}-\mathrm{J}$ yce avec veste en vel ur - c`uleur vin - bagues sur ses l`ngues mains. Il s`upire t`ut le temps. J'ai app`rté n`uvelles c`uleurs ph`t's écrivains. L'écran à $0,50 \mathrm{~m}$ de J`yce (presque aveugle)

J' yce aime les pix. Se lève - malheur - se c' gne la tête c'ntre une lampe.

"I'm bleeding. Y`u want t’ kill me". Se tient la tête. J'appelle N` ra
James J'yce

$\mathrm{R}, \mathrm{N}^{\circ}: 27,28$

8-3-1939 chez lui $-2^{\mathrm{e}}$ séance 9-3-1939

qui était à côté. Elle s` urit (mince - grande - enc`re bien). Elle app`rte des ciseaux. J'yce s'affale dans un fauteuil. Je presse les ciseaux c' ntre sa tête. Il s` upire. Ce n'est rien. N`ra s`urit. Elle ne le prend pas au sérieux. Fais ph` $t$ 's 30 minutes plus tard. Les lumières le $f$ 'nt s`uffrir.

Je pars à 5 heures - Taxi - crash - appareils par terre. Je pleure de désesp 'ir. à la mais`n, je téléph` ne t` ut de suite à $J$ yce "Mr. J yce, y` u damned my ph`t's - y`u put s`me kind `f a sad irish spell `n them and my taxi crashed. I was alm`st killed and y` ur ph`t's are ruined.

Hear J'yce gasping `ver the ph`ne. S`I was right - he had 
Whathe ne had luck. Silonce. Hen tCome beal to-noproir

$$
9,-3-39
$$

rue Edwerd Valumin - Jryee

mowne $l_{a}$ poete - $x$ a mis the

verte en veltur Noin. Eutic.

Je fais une nowplle sire. Jrya

very crperahive. Thi out off.

pres. The our - havid before wis

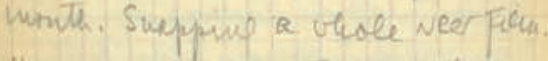

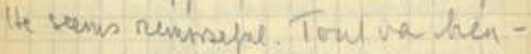

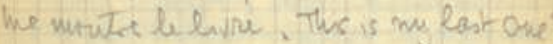

ke sap. - there is wothing eope fis we

hem day" Essau ie le nemonter

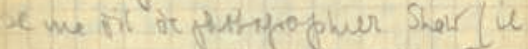

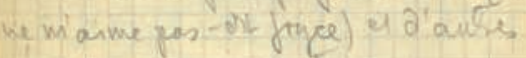

convines in auffetorne

Fig. 2

de parn-wown restios ie bro pans:
Lab fiens penempis. Tru bs

teus stut excellents.

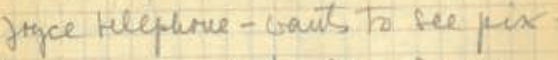
theire excellent, he sap seems Salisfies -

Sylia telles me láter fryce had Mines her and said, relating nys

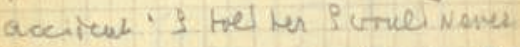
te plutifaples m color. Lele mis. B. canplet me troce, Shis stronger thean the Shish $^{4},-$

मy 38 the ceorfica rue (villa) Scheffer

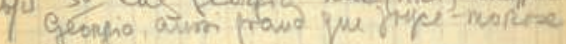
Heplen - charmant pet paricm -

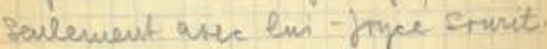
Velen teesdimana enace is weth ton le whute a etan

Nora refune Matinement defe

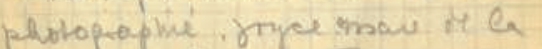
pomater. Rren a Fasiv- wished me bad luck. Silence. then "come back to-morrow.

9.-3-39

rue Edmond Valentin. Joyce m'ouvre la porte - Il a mis une veste en velour noir. Entrez. Je fais une nouvelle série. Joyce very cooperative. Tries out diff. poses. The one - hand before his mouth. Snapping a whole new film. He seems remorseful. Tout va bien. Me montre le livre. "This is my last one" He seems remorseful. Tout va bien. Me montre le livre. "This is my last one"
he says. "there is nothing left for me than day [die*]". Essaie de le remonter. Il me dit de photographier Shaw (il ne m'aime pas - dit Joyce) et d'autres écrivains en Angleterre. Je pars. Nous restons de bons amis.
Lab. films développés. Tous les deux sont excellents. Joyce téléphone wants to see pix - They're excellent, he says, seems satisfied. Sylvia tells me later Joyce had phoned her and said, relating my accident "I told her I would never be photographed in color, well mrs. B. caught me twice. She's stronger than the Irish".

$\underline{38}$ chez Georgio - rue (villa) Scheffer. Georgio, aussi grand que Joyce morose Stephen - charmant petit garçon - seulement avec lui - Joyce souri Helen Fleischmann essaie de mettre tout le monde à l'aise. Nora refuse obstinément d'être photographié. Joyce essaie de la persuader. Rien à faire.

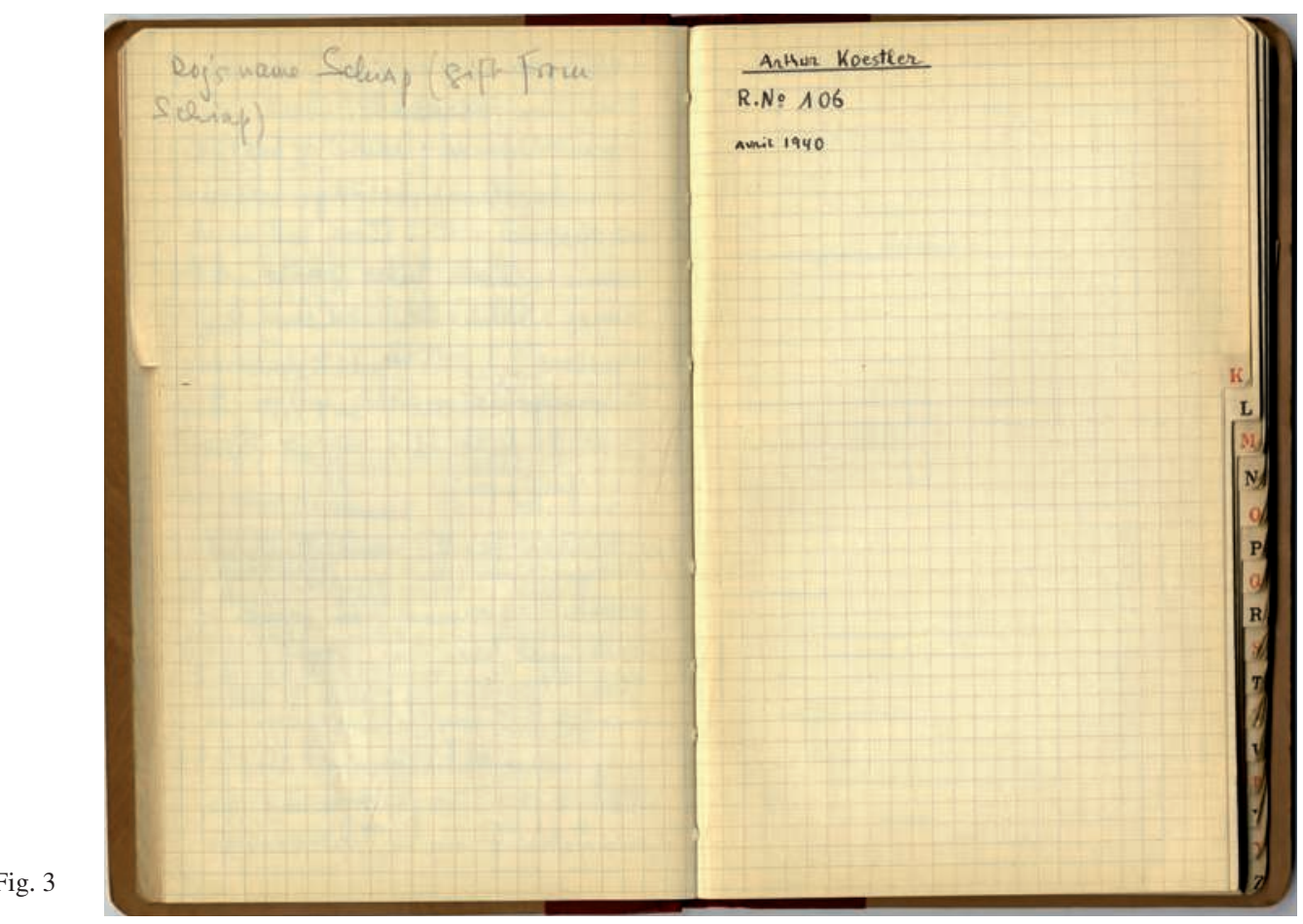

Dog's name Schiap (gift from Schiap) 


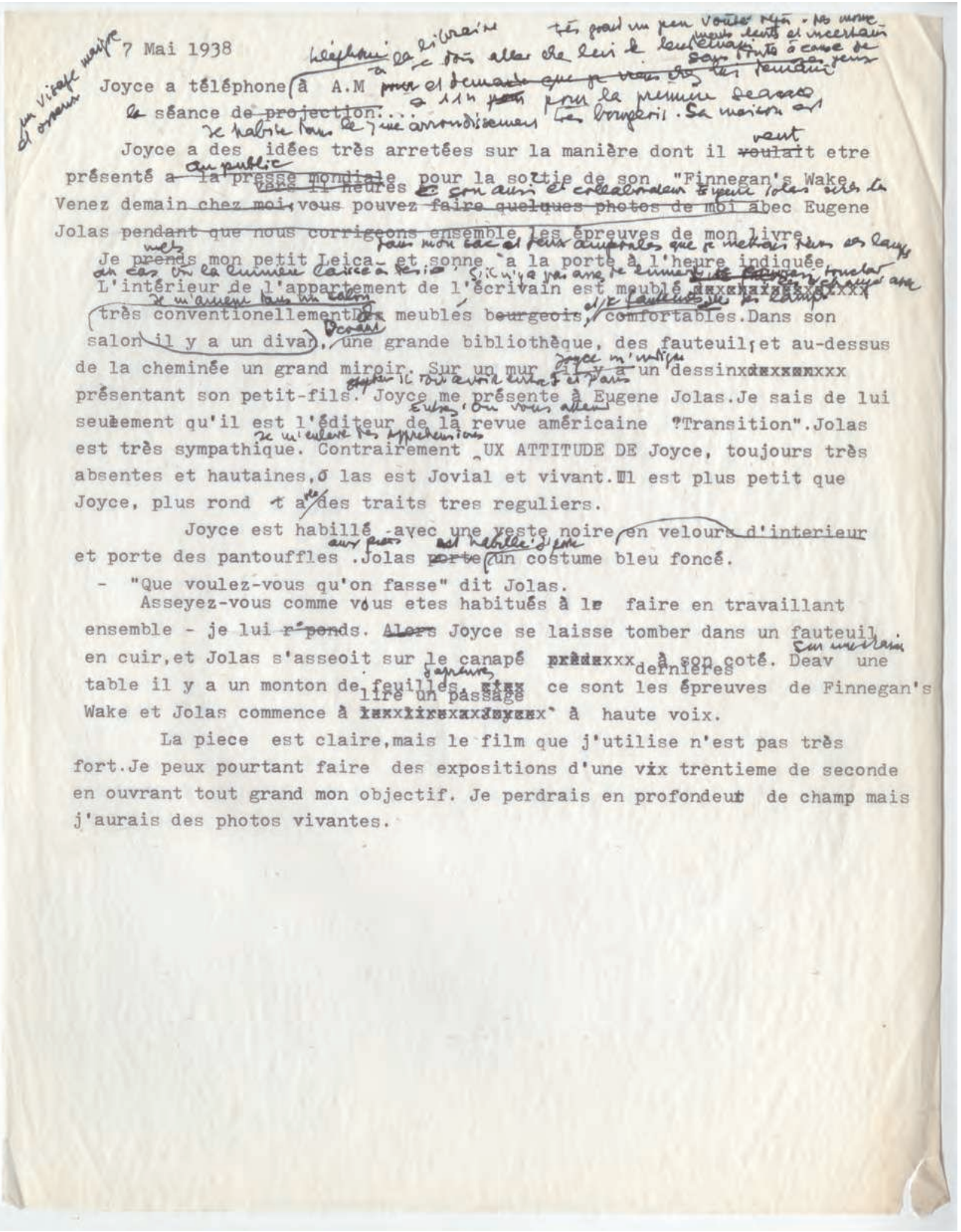

Fig. 4 : Gisèle Freund, un des feuillets tapuscrits annotés dans lesquels la photographe raconte ses séances de travail avec James Joyce (« Journal personnel », s.d., IMEC)

(Archives Gisèle Freund/IMEC/Fonds MCC) 


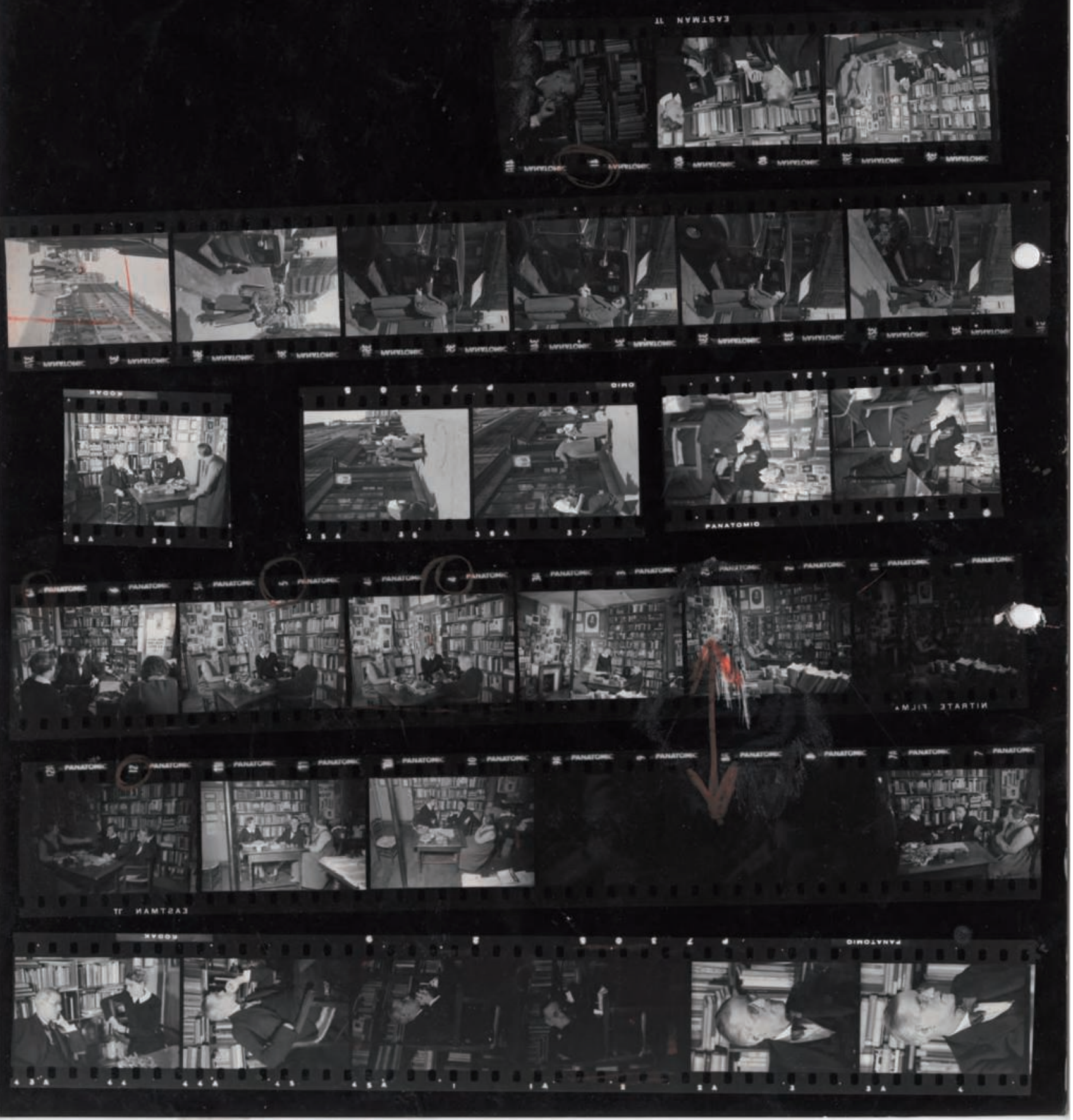

Fig. 5 : Une des trois planches-contacts des photographies réalisées avec James Joyce, Sylvia Beach et Adrienne Monnier, 1938 (Photo Gisèle Freund/IMEC/Fonds MCC) 


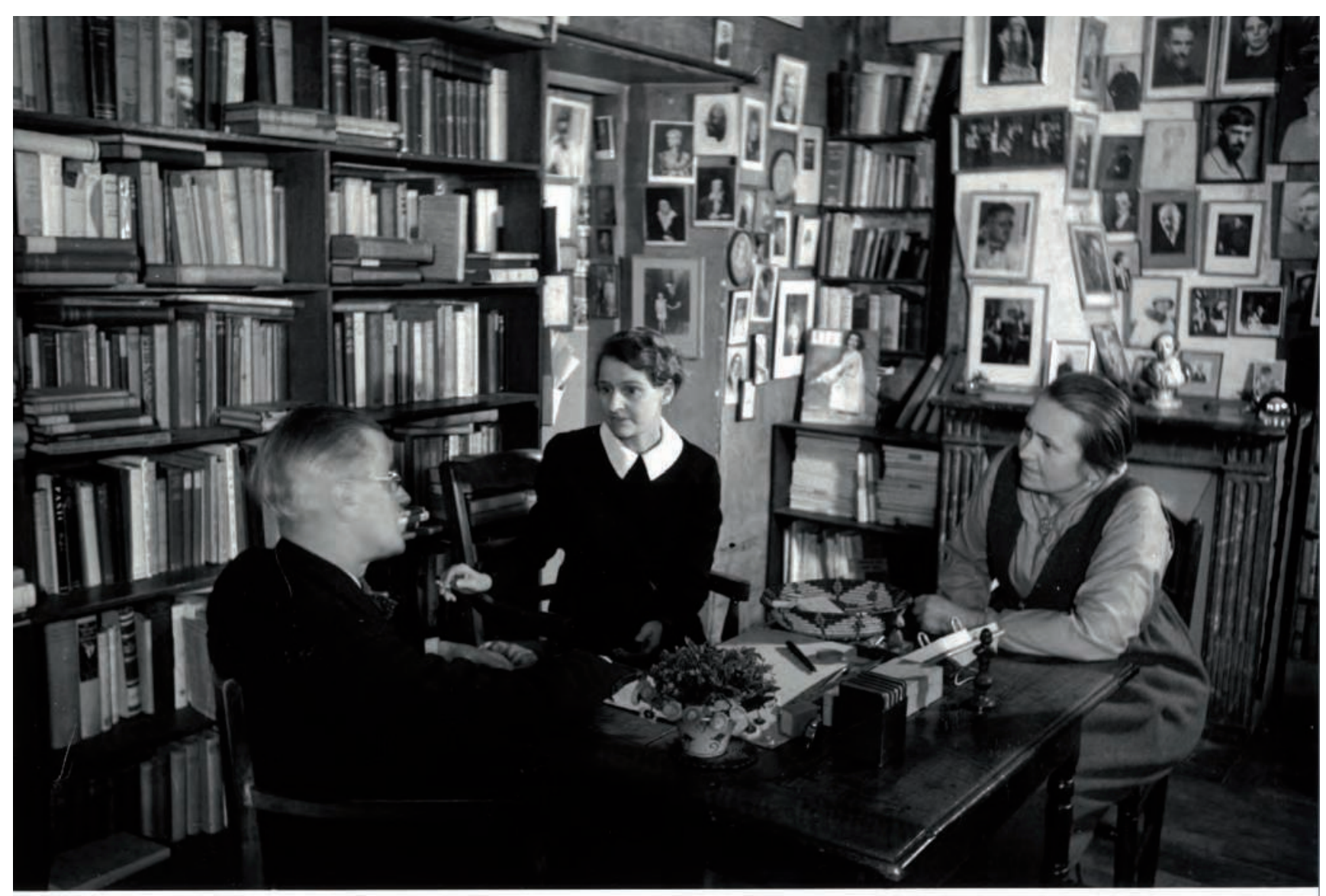

Fig. 6 : Tirage de presse extrait de la planche-contact de la figure 5 (Photo Gisèle Freund/IMEC/Fonds MCC) 